EUROPEAN JOURNAL OF PURE AND APPLIED MATHEMATICS

Vol. 11, No. 1, 2018, 138-149

ISSN 1307-5543 - www.ejpam.com

Published by New York Business Global

\title{
Littlewood-Paley $g$-function and Radon transform on the Heisenberg group
}

\author{
Zheng Fang ${ }^{1}$, Jianxun $\mathrm{He}^{1, *}$ \\ ${ }^{1}$ School of Mathematics and Information Sciences, Guangzhou University, Guangzhou 510006, \\ China
}

\begin{abstract}
In this paper, we consider Radon transform on the Heisenberg group $\mathbf{H}^{n}$, and obtain new inversion formulas via dual Radon transforms and Poisson integrals. We prove that the Radon transform is a unitary operator from Sobelov space $W$ into $L^{2}\left(\mathbf{H}^{n}\right)$. Moreover, we use the Radon transform to define the Littlewood-Paley $g$-function on a hyperplane and obtain the LittlewoodPaley theory.
\end{abstract}

2010 Mathematics Subject Classifications: 44A12; 43A15

Key Words and Phrases: Heisenberg group, Little-wood Pelay $g$-function, Radon transform

\section{Introduction}

We identify any point $(x, y)$ in $\mathbb{R}^{2 n}$ with the point $z=x+i y$ in $\mathbb{C}^{n}$ and denote the symplectic form $[\cdot, \cdot]$ on $\mathbb{C}^{n}$ by $[z, w]=\frac{1}{2} \operatorname{Im}\langle z, w\rangle$ for $z, w \in \mathbb{C}^{n}$. We define the multiplication on $\mathbb{C}^{n} \times \mathbb{R}$ by

$$
(z, t)\left(z^{\prime}, t^{\prime}\right)=\left(z+z^{\prime}, t+t^{\prime}+\frac{1}{2} \operatorname{Im}\left\langle z, z^{\prime}\right\rangle\right)
$$

for all $(z, t)$ and $\left(z^{\prime}, t^{\prime}\right)$ in $\mathbb{C}^{n} \times \mathbb{R}$. The group $\mathbb{C}^{n} \times \mathbb{R}$ with respect to the multiplication defined by (1) is denoted by $\mathbf{H}^{n}$ and is called the Heisenberg group. It is well known that the Heisenberg group plays an important role in several branches of mathematics. There are, therefore, several ways of realising the group due to the widely application of the Heisenberg group (see $[2,17]$ ). In 1917, Radon proved that a smooth function in $\mathbb{R}^{3}$ is completely determined by its integrals over all the planes. This leads in a more general setting to the consideration of the Radon transform. The research of Radon transform has made important influence due to its wide applications to partial differential equations, $X$-ray technology, radio astronomy and so on. The basic theory and some new results can be found in [9]. Geller-Stein [5] and Strichartz [16] introduced the Heisenberg-Radon

${ }^{*}$ Corresponding author.

Email addresses: fangzheng@e.gzhu.edu.cn (Z. Fang), hejianxun@gzhu.edu.cn (J. He) 
transform on $\mathbf{H}^{n}$. He-Liu considered inversion formulas of the Radon transform on $\mathbf{H}^{n}$ and Siegel type Lie group in $[6,7,8]$ by using the continuous wavelet transforms. The combination of Radon transform and wavelet transform has proved to be very useful both in pure mathematics and applied science. Therefore, it is very meaningful to give the inversion formula the Radon transform by using various ways. In addition, because of great application of the Heisenberg group, many scholars have studied Littlewood-Paley theory on the Heisenberg group in recent years. Thangavelu [17] studied the $g$-function connected with the semigroup generated by the sub-Laplacian. Liu-Ma [12] investigated the $g$-function related to a class of radial functions in which the characterization of the $L^{p}\left(\mathbf{H}^{n}\right)$-norm of a function on $\mathbf{H}^{n}$ was obtained.

This paper is organized as follows. In section 2, we recall some notations, definitions and preliminary fact. Motivated by [5, 9, 10], we mainly introduce singular convolution operators on $\mathbf{H}^{n}$ and obtain inverse formulas of Radon transform in section 3. Section 4 is to define Littlewood-Paley $g$-function in some hyperplanes and establishes LittlewoodPaley theory, which generalizes the results in [12]. At last, in section 5 we show that the Radon transform and the Poisson integral for the Šliov boundary are equivalent, and the inverse formula is given by the classical Schwarz theorem.

\section{Preliminaries}

We denote by $\mathscr{O}$ the space all holomorphic functions on $\mathbb{C}^{n}$, the Fock space with $\lambda \in(0, \infty)$ is defined by

$$
\mathscr{H}_{\lambda}:=\left\{f \in \mathscr{O}:\|f\|^{2}:=\int_{\mathbb{C}^{n}}|f(w)|^{2} e^{-\pi \lambda|w|^{2}} \lambda^{n} d w<\infty\right\}
$$

and $\mathscr{H}_{-\lambda}:=\left\{\bar{f}: f \in \mathscr{H}_{\lambda}\right\}$. The scalar product is given by

$$
\langle f, g\rangle:=\int_{\mathbb{C}^{n}} f(w) \bar{g}(w) e^{-\pi \lambda|w|^{2}} \lambda^{n} d w .
$$

Now, an arbitrary complete orthonormal system of functions $\psi_{\lambda, \alpha} \in \mathscr{H}_{\lambda}$ on $\mathbb{C}^{n}$ is represented by

$$
\psi_{\lambda, \alpha}(w)=\frac{\lambda^{|\alpha| / 2} w^{\alpha}}{\left(2^{|\alpha|} \alpha !\right)^{1 / 2}}=\frac{w_{1}^{\alpha_{1}}}{\left((2 / \lambda)^{\alpha_{1}} \alpha_{1} !\right)^{1 / 2}} \cdots \frac{w_{n}^{\alpha_{n}}}{\left((2 / \lambda)^{\alpha_{n}} \alpha_{n} !\right)^{1 / 2}}
$$

which satisfies

$$
\left\langle\psi_{\lambda, \alpha}, \psi_{\lambda, \beta}\right\rangle=\delta_{\alpha \beta}
$$

where $\delta_{\alpha \beta}$ denotes the Kronecker symbol and $\alpha=\left(\alpha_{1}, \ldots, \alpha_{n}\right) \in \mathbb{N}^{n}$. It is natural that $f \in \mathscr{H}_{\lambda}$ is represented by a series

$$
f(w)=\sum_{\alpha \in \mathbf{N}^{n}}\left\langle f, \psi_{\lambda, \alpha}\right\rangle \psi_{\lambda, \alpha}
$$


We define the Bargmann-Fock representation $\pi_{\lambda}$ from $\mathbf{H}^{n}$ into the group $G$ of all unitary operators on $\mathscr{H}_{\lambda}$ by, for any $\psi \in \mathscr{H}_{ \pm \lambda}$ and any $(z, t) \in \mathbf{H}^{n}$,

$$
\pi_{\lambda}(z, t) \psi(w)=e^{-2 \pi i \lambda t+\pi \lambda\langle w, z\rangle-\pi \lambda|z|^{2} / 2} \psi(w-z),
$$

and

$$
\pi_{-\lambda}(z, t) \psi(w)=e^{2 \pi i \lambda t+\pi \lambda\langle z, w\rangle-\pi \lambda|z|^{2} / 2} \psi(w-z),
$$

for any $\lambda \in \mathbb{R}^{*}:=\mathbb{R} \backslash\{0\}$. It is easy to see that $\pi_{\lambda}: \mathbf{H}^{n} \rightarrow G$ is a group homomorphism and $\pi_{\lambda}(z, t) \psi \rightarrow \psi$ in $\mathscr{H}_{\lambda}$ as $(z, t) \rightarrow(0,0)$. Then the unitary representation $\pi_{\lambda}$ of $\mathbf{H}^{n}$ on $\mathscr{H}_{\lambda}$ is irreducible in the sense that the only closed subspaces of $\mathscr{H}_{\lambda}$ that are invariant under all the operators $\pi_{\lambda}(z, t),(z, t) \in \mathbf{H}^{n}$, are $\{0\}$ and $\mathscr{H}_{\lambda}$. Two unitary representations $\pi_{\lambda}$ and $\pi_{\mu}$ of $\mathbf{H}^{n}$ are unitarily equivalent if and only if $\lambda=\mu$. Now, we are able to introduce the following notion of the group Fourier transform on $\mathbf{H}^{n}$. Let $f \in L^{1}\left(\mathbf{H}^{n}\right)$. Then the group Fourier transform $\pi_{\lambda}$ on $f$ is defined by

$$
\pi_{\lambda}(f)=\int_{\mathbf{H}^{n}} f(z, t) \pi_{\lambda}(z, t) d z d t
$$

Thus, the Plancherel theorem states as follows.

Lemma 1. Let $f \in L^{2}\left(\boldsymbol{H}^{n}\right)$. Then

$$
\|f\|_{L^{2}\left(\boldsymbol{H}^{n}\right)}^{2}=\int_{\mathbb{R}^{*}}\left\|\pi_{\lambda}(f)\right\|_{H S}^{2}|\lambda|^{n} d \lambda .
$$

And the inversion formula is valid:

$$
f(z, t)=\int_{\mathbb{R}^{*}} \operatorname{tr}\left(\pi_{\lambda}^{*}(f) \pi_{\lambda}(z, t)\right)|\lambda|^{n} d \lambda
$$

\section{The inverse formulas of Radon transforms}

In this section, our purpose is to get the inverse formulas of Radon transforms. In order to do this we first recall the Abel Fourier transform.

The Fourier transform for $t$ and $z$-variable, respectively, are given by

$$
\mathscr{F}_{2} f(z, t)=\int_{\mathbb{R}} f\left(z, t^{\prime}\right) e^{-2 \pi i t^{\prime} t} d t^{\prime},
$$

and

$$
\mathscr{F}_{1} f(z, t)=\int_{\mathbb{C}^{n}} f\left(z^{\prime}, t\right) e^{-2 \pi i \operatorname{Re}\left\langle z, z^{\prime}\right\rangle} d z^{\prime}
$$

The symplectic Fourier transform on $\mathbb{C}^{n}$ is defined by, for any $g \in L^{2}\left(\mathbb{C}^{n}\right)$,

$$
\mathscr{F}_{s} g(z)=\int_{\mathbb{C}^{n}} g\left(z^{\prime}\right) e^{\pi i \operatorname{Im}\left\langle z, z^{\prime}\right\rangle} d z^{\prime},
$$


and then we also obtain

$$
\mathscr{F}_{s} g(z)=\mathscr{F}_{1} g(i z / 2) .
$$

Let $\pi_{\lambda}(f)$ be as in $(3)$ and $\pi_{\lambda}(z, 0):=\pi_{\lambda}(z)$. Then we define

$$
\pi_{\lambda}(f)=\int_{\mathbb{C}^{n}} \mathscr{F}_{2} f(z, \lambda) \pi_{\lambda}(z) d z
$$

Therefore, the integral operator $w_{\lambda}$ for $g$ is defined by

$$
w_{\lambda}(g)=\int_{\mathbb{C}^{n}} g(z) \pi_{\lambda}(z) d z
$$

and Plancherel formula is given by

$$
\left\|w_{\lambda}(g)\right\|_{H S}^{2}=\lambda^{-n} \int_{\mathbb{C}^{n}}|g(z)|^{2} d z .
$$

Next, to introduce the notion of the singular convolution operator on $\mathbf{H}^{n}$, we need the following notation. Let $T^{n}=\left(\frac{\partial}{\partial t}\right)^{n}$. We consider hyperplanes

$$
\mathscr{E}_{(z, t)}:=\left\{\left(z, t+\frac{1}{2} \operatorname{Im}\left\langle z, z^{\prime}\right\rangle\right) \text { for any } z^{\prime} \in \mathbb{C}^{n}\right\}
$$

and

$$
\mathscr{E}_{\left(z^{\prime}, t^{\prime}\right)}^{*}:=\left\{\left(z^{\prime}, t^{\prime}-\frac{1}{2} \operatorname{Im}\left\langle z, z^{\prime}\right\rangle\right) \text { for any } z \in \mathbb{C}^{n}\right\} .
$$

As we know, $T^{n}$ is studied in fractional differential equations due to its wide applications (see $[1,14,15])$. In this paper, Combining with these hyperplanes and $T^{n}$ we have the following definition.

Definition 1. The singular convolution operator $R_{n}$ and the dual singular convolution operator $R_{n}^{t}$ for function $f, \phi$, respectively, are defined by

$$
R_{n}(f)(z, t)=\int_{\mathbb{C}^{n}} T^{n} f\left(z^{\prime}, t+\frac{1}{2} \operatorname{Im}\left\langle z, z^{\prime}\right\rangle\right) d z^{\prime}
$$

and

$$
R_{n}^{t} \phi\left(z^{\prime}, t^{\prime}\right)=\int_{\mathbb{C}^{n}} T^{n} \phi\left(z, t^{\prime}-\frac{1}{2} \operatorname{Im}\left\langle z, z^{\prime}\right\rangle\right) d z .
$$

In particular, $R_{0}$ is the Heisenberg Radon transform when $n=0$ (see [16]). It is easy to see that $R_{n}^{t} \phi=R_{n} \phi$ (also see [4]). We denote by $\mathscr{S}\left(\mathbf{H}^{n}\right)$ the space all Schwartz functions on $\mathbf{H}^{n}$. Using (9) and (10), we have the following identity

$$
\int_{\mathbf{H}^{n}} f(z, t) R_{n}^{t} \phi(z, t) d z d t=\int_{\mathbf{H}^{n}} R_{n} f(z, t) \phi(z, t) d z d t
$$

for any $f, \phi \in \mathscr{S}\left(\mathbf{H}^{n}\right)$. 
Proposition 1. Let $f \in \mathscr{S}\left(\boldsymbol{H}^{n}\right)$. Then, for any $\lambda \in \mathbb{R}^{*}$, we have

$$
\pi_{\lambda}\left(R_{n} f(z, t)\right)=(2 \pi i \lambda)^{n} w_{\lambda}(\mathscr{F} f(i \lambda z / 2, \lambda)) .
$$

Proof. Let $\varphi_{\lambda, \alpha}$ be as in (2) and differential operator $T^{n}$. As we know,

$$
\mathscr{F}_{2}\left(T^{n} f\right)(\lambda)=(2 \pi i \lambda)^{n} \mathscr{F}_{2}(f)(\lambda) .
$$

According to the (7), (11), together with (4) and (5), we have

$$
\begin{aligned}
{\left[\pi_{\lambda}\left(R_{n} f\right) \varphi_{\lambda, \alpha}\right] } & =\left[w_{\lambda} \mathscr{F}_{2}\left(R_{n} f(z, \lambda)\right) \varphi_{\lambda, \alpha}(w)\right] \\
& =\int_{\mathbb{C}^{n}} \mathscr{F}_{2}\left(R_{n} f(z, \lambda)\right)\left[\pi_{\lambda}(z) \varphi_{\lambda, \alpha}(w)\right] d z \\
& =\int_{\mathbb{C}^{n}} \int_{\mathbb{R}} R_{n} f(z, t) e^{-2 \pi i \lambda t}\left[\pi_{\lambda}(z) \varphi_{\lambda, \alpha}(w)\right] d z d t \\
& =(2 \pi i \lambda)^{n} \int_{\mathbb{C}^{n}} \int_{\mathbb{R}} \int_{\mathbb{C}^{n}} f\left(z^{\prime}, t\right) e^{-2 \pi i \lambda t} e^{\pi i \lambda \operatorname{Im}\left\langle z, z^{\prime}\right\rangle}\left[\pi_{\lambda}(z) \varphi_{\lambda, \alpha}\right] d z^{\prime} d z d t \\
& =(2 \pi i \lambda)^{n} \int_{\mathbb{C}^{n}} \int_{\mathbb{C}^{n}} \mathscr{F}_{2} f\left(z^{\prime}, \lambda\right) e^{\pi i \lambda \operatorname{Im}\left\langle z, z^{\prime}\right\rangle}\left[\pi_{\lambda}(z) \varphi_{\lambda, \alpha}(w)\right] d z^{\prime} d z \\
& =(2 \pi i \lambda)^{n} \int_{\mathbb{C}^{n}} \mathscr{F}_{2} \mathscr{F}_{s} f(\lambda z, \lambda)\left[\pi_{\lambda}(z) \varphi_{\lambda, \alpha}(w)\right] d z .
\end{aligned}
$$

In addition, we need the full Euclidean Fourier transform

$$
\mathscr{F} f(z, t)=\int_{\mathbb{R}} \int_{\mathbb{C}^{n}} f\left(z^{\prime}, t^{\prime}\right) e^{-2 \pi i t t^{\prime}} e^{-2 \pi i \operatorname{Re}\left\langle z, z^{\prime}\right\rangle} d z^{\prime} d t^{\prime} .
$$

By this and (5), we find that

$$
\begin{aligned}
{\left[\pi_{\lambda}\left(R_{n} f\right) \varphi_{\lambda, \alpha}(w)\right] } & =(2 \pi i \lambda)^{n} \int_{\mathbb{C}^{n}} \mathscr{F} f(i \lambda z / 2, \lambda)\left[\pi_{\lambda}(z) \varphi_{\lambda, \alpha}(w)\right] d z \\
& =(2 \pi i \lambda)^{n}\left[w_{\lambda}(\mathscr{F} f(i \lambda z / 2, \lambda)) \varphi_{\lambda, \alpha}(w)\right] .
\end{aligned}
$$

The proof is completed.

Proposition 2. Let $\phi \in \mathscr{S}\left(\boldsymbol{H}^{n}\right)$. Then

$$
(8 \pi)^{n}(i \lambda)^{-n} \mathscr{F}_{2} \phi(z / \lambda, \lambda)=\mathscr{F}_{s} \mathscr{F}_{2}\left[R_{n}^{t} \phi(z, \lambda)\right]=\mathscr{F}\left[R_{n}^{t} \phi(i z / 2, \lambda)\right] .
$$

Proof. Let $\phi \in \mathscr{S}\left(\mathbf{H}^{n}\right)$, By (10), (11) and (6), we deduce that

$$
\begin{aligned}
\mathscr{F}_{s} \mathscr{F}_{2}\left[R_{n}^{t} \phi(z, \lambda)\right] & =(2 \pi i \lambda)^{n} \int_{\mathbb{C}^{n}} \int_{\mathbb{R}} \int_{\mathbb{C}^{n}} \phi\left(w, t-\frac{1}{2} \operatorname{Im}\left\langle w, z^{\prime}\right\rangle\right) e^{-2 \pi i \lambda t} e^{\pi i \operatorname{Im}\left\langle z, z^{\prime}\right\rangle} d w d z^{\prime} d t \\
& =(2 \pi i \lambda)^{n} \int_{\mathbb{C}^{n}} \int_{\mathbb{C}^{n}} \mathscr{F}_{2} \phi(w, \lambda) e^{\pi i \operatorname{Im}\left\langle z, z^{\prime}\right\rangle} e^{-\pi \lambda i \operatorname{Im}\left\langle w, z^{\prime}\right\rangle} d w d z^{\prime}
\end{aligned}
$$




$$
\begin{aligned}
& =(2 \pi i \lambda)^{n} \int_{\mathbb{C}^{n}} \int_{\mathbb{C}^{n}} \mathscr{F}_{2} \phi(w, \lambda) e^{\pi i \operatorname{Im}\left\langle z, z^{\prime}\right\rangle} e^{\pi \lambda i \operatorname{Im}\left\langle z^{\prime}, w\right\rangle} d w d z^{\prime} \\
& =(2 \pi i \lambda)^{n} \int_{\mathbb{C}^{n}} \mathscr{F}_{2} \mathscr{F}_{s} \phi\left(\lambda z^{\prime}, \lambda\right) e^{\pi i \operatorname{Im}\left\langle z, z^{\prime}\right\rangle} d z^{\prime} \\
& =(2 \pi i \lambda)^{n} \int_{\mathbb{C}^{n}} \mathscr{F}_{1} \mathscr{F}_{2} \phi\left(i \lambda z^{\prime} / 2, t\right) e^{\pi i \operatorname{Im}\left\langle z, z^{\prime}\right\rangle} d z^{\prime} \\
& =(2 \pi i \lambda)^{n} \int_{\mathbb{C}^{n}} \mathscr{F}_{1} \mathscr{F}_{2} \phi\left(i \lambda z^{\prime} / 2, \lambda\right) e^{2 \pi i \operatorname{Re}\left\langle z / \lambda, i \lambda z^{\prime} / 2\right\rangle} d z^{\prime} \\
& =(8 \pi)^{n}(i \lambda)^{-n} \mathscr{F}_{2} \phi(z / \lambda, \lambda) \text {. }
\end{aligned}
$$

This finishes the proof of Proposition 2.

Theorem 1. Let $f \in \mathscr{S}\left(\boldsymbol{H}^{n}\right) \cap L^{2}\left(\boldsymbol{H}^{n}\right)$ and $\phi(z, t):=R_{n} f(z, t) \in \mathscr{S}\left(\boldsymbol{H}^{n}\right)$. Then $f(z, t)=$ $(4 \pi)^{-2 n} R_{n}^{t} \phi(z, t)$ holds in $L^{2}\left(\boldsymbol{H}^{n}\right)$.

Proof. According to the inversion formula in Lemma 1 and (8), we obtain

$$
\begin{aligned}
\int_{\mathbf{H}^{n}}\left|R_{n} f(z, t)\right|^{2} d z d t & =\int_{\mathbb{R}^{*}}\left\|\pi_{\lambda}\left(R_{n} f\right)\right\|_{H S}^{2}|\lambda|^{n} d \lambda \\
& =\int_{\mathbb{R}^{*}}\left\|(2 \pi i \lambda)^{n} w_{\lambda}(\mathscr{F} f(i \lambda z / 2, \lambda))\right\|_{H S}^{2}|\lambda|^{n} d \lambda \\
& =\int_{\mathbb{R}^{*}} \int_{\mathbb{C}^{n}}(4 \pi)^{2 n}(\lambda)^{-n}|\mathscr{F} f(z, \lambda)|^{2} d z|\lambda|^{n} \mid d \lambda \\
& =\int_{\mathbb{R}^{*}}(4 \pi)^{2 n}\left\|w_{\lambda}(\mathscr{F} f(z, \lambda))\right\|_{H S}^{2}|\lambda|^{n} d \lambda \\
& =\int_{\mathbb{R}^{*}}(4 \pi)^{2 n}\left\|\pi_{\lambda} \mathscr{F}_{1} f(\cdot, \lambda)\right\|_{H S}^{2}|\lambda|^{n} d \lambda \\
& =\int_{\mathbf{H}^{n}}(4 \pi)^{2 n}\left|\mathscr{F}_{1} f(z, t)\right|^{2} d z d t,
\end{aligned}
$$

which implies that,

$$
(4 \pi)^{n} \mathscr{F}_{1} f(z, t)=\int_{\mathbb{R}^{*}} \operatorname{tr}\left(\pi_{\lambda}^{*}\left(R_{n} f\right) \pi_{\lambda}(z, t)\right)|\lambda|^{n} d \lambda=R_{n} f(z, t)
$$

and hence

$$
f(z, t)=(4 \pi)^{-n} \mathscr{F}_{1}^{-1} R_{n} f(z, t)
$$

From (6), it follows that

$$
\int_{\mathbf{H}^{n}}\left|\mathscr{F}_{1} \mathscr{F}_{2} R_{n}^{t} \phi(i z / 2, \lambda)\right|^{2} d z d \lambda=4^{n} \int_{\mathbf{H}^{n}}\left|\mathscr{F}_{1} \mathscr{F}_{2} R_{n}^{t} \phi(z, \lambda)\right|^{2} d z d \lambda
$$

By Proposition 2, we find that

$$
4^{n} \int_{\mathbf{H}^{n}}\left|\mathscr{F}_{1} \mathscr{F}_{2} R_{n}^{t} \phi(z, \lambda)\right|^{2} d z d \lambda=(8 \pi)^{2 n}(i \lambda)^{-2 n} \int_{\mathbf{H}^{n}}\left|\mathscr{F}_{2} \phi(z / \lambda, \lambda)\right|^{2} d z d \lambda
$$




$$
=(8 \pi)^{2 n} \int_{\mathbf{H}^{n}}\left|\mathscr{F}_{2} \phi(z, \lambda)\right|^{2} d z d \lambda,
$$

which implies that,

$$
\mathscr{F}_{1} \mathscr{F}_{2} R_{n}^{t} \phi(z, \lambda)=(4 \pi)^{n} \mathscr{F}_{2} \phi(z, \lambda)=(4 \pi)^{n} \mathscr{F}_{2} R_{n} f(z, \lambda)
$$

holds in $L^{2}\left(\mathbf{H}^{n}\right)$. Combining with (12) and (13), we have

$$
f(z, t)=(4 \pi)^{-2 n} R_{n}^{t} \phi(z, t) .
$$

We complete the proof of Theorem 1 .

The Sobolev space $W$ on $\mathbf{H}^{n}$ is defined by

$$
W:=\left\{f \in L^{2}\left(\mathbf{H}^{n}\right): \int_{\mathbf{H}^{n}}\left|T^{n} f(z, t)\right|^{2} d z d t<\infty\right\} .
$$

Theorem 2. Let $f \in W$. Then there exists a constant $C_{n}$ such that $T^{n} R_{0}^{t} T^{n} R_{0} f=C_{n} f$ holds in $L^{2}\left(\boldsymbol{H}^{n}\right)$.

Proof. Let $f \in W \cap L^{2}\left(\mathbf{H}^{n}\right)$, it is easy to verify that

$$
R_{0}\left(T^{n}(f)\right)=T^{n}\left(R_{0}(f)\right) .
$$

By Lemma 1 and the proof of Theorem 1, we find that

$$
\int_{\mathbb{C}^{n}}\left|R_{0} T^{n} f(z, t)\right|^{2} d z d t=\int_{\mathbb{R}^{*}}\left\|\pi_{\lambda}\left(R_{n}(f)\right)\right\|_{H S}^{2}|\lambda|^{n} d \lambda=(4 \pi)^{2 n}\|f\|_{L^{2}\left(\mathbf{H}^{n}\right)}^{2} .
$$

Together with the density argument, we obtain that (14) holds in $W \cap \mathscr{S}\left(\mathbf{H}^{n}\right)$.

Similarly, we also have

$$
R_{0}^{t} T^{n} \phi=T^{n} R_{0}^{t} \phi
$$

Obviously, $C_{n} R_{0} T^{n}(f)=\phi \in W$ is well defined. According to (15) and (14), we see that

$$
R_{0}^{t} T^{n} \phi=T^{n} R_{0}^{t} \phi=C_{n} T^{n} R_{0}^{t} T^{n} R_{0} f=C_{n} \widetilde{R}_{0}^{t} R_{0} f=C_{n} f .
$$

We remark that the inverse formula of Radon transform is obtained via Propositions 1, 2 and Theorems 1, 2.

\section{Littlewood-Paley $g$-function}

Let $X_{j}=\frac{\partial}{\partial x_{j}}+\frac{1}{2} y_{j} \frac{\partial}{\partial t}, Y_{j}=\frac{\partial}{\partial y_{j}}-\frac{1}{2} x_{j} \frac{\partial}{\partial t}, j=1, \ldots, n . X_{j}, Y_{j}$ be left invariant vector fields on $\mathbf{H}^{n}$. The gradient operator on $\mathbf{H}^{n}$ is given by

$$
\nabla=\left(X_{1}, \ldots, X_{n}, Y_{1}, \ldots, Y_{n}\right)
$$


The sub-Laplacian operator of $\mathbf{H}^{n}$ is defined by

$$
\mathscr{L}=\sum_{j=1}^{n}\left(X_{j}^{2}+Y_{j}^{2}\right) .
$$

It is known that

$$
\pi_{\lambda}(\mathscr{L} f)(\lambda)=\pi_{\lambda}(f) \sum_{\alpha \in \mathbb{N}^{n}}(2|\alpha|+n)|\lambda| \phi_{\lambda, \alpha}
$$

$\mathscr{L}$ is a positive self-adjoint operator. For a suitable function $\psi$ defined on $(0, \infty)$, the operator $\psi(\mathscr{L})$ can be defined in terms of the spectral expansion of $\mathscr{L}$. Then

$$
\pi_{\lambda}(\psi(\mathscr{L}) f)(\lambda)=\pi_{\lambda}(f) \sum_{\alpha \in \mathbb{N}^{n}} \psi(2|\alpha|+n)|\lambda| \phi_{\lambda, \alpha}
$$

Let $\phi$ denote the kernel function of $\psi(\mathscr{L})$. Then

$$
\phi(\lambda)=\sum_{\alpha \in \mathbb{N}^{n}} \psi(2|\alpha|+n)|\lambda| \phi_{\lambda, \alpha}
$$

We denote by $\mathscr{R}\left(\mathbf{H}^{n}\right)$ the set of all these functions $\phi$.

Let $\phi \in \mathscr{R}\left(\mathbf{H}^{n}\right)$, the Littlewood-Paley $g$-function on $\mathbf{H}^{n}$ is defined by

$$
g\left(R_{n} f, u\right)=\left[\int_{0}^{\infty}\left|R_{n} f * \phi_{\rho}(u)\right|^{2} \frac{d \rho}{\rho}\right]^{1 / 2},
$$

where $R_{n} f$ is as in (9) and $\phi_{\rho}(u)=\rho^{-n-1} \phi\left(\frac{u}{\sqrt{\rho}}\right)$ for all $\rho>0$.

The homogeneous norm on the Heisenberg group is given by $|u|=|(z, t)|=\left(|z|^{4}+\right.$ $\left.t^{2}\right)^{1 / 4}$, which satisfies the trigonometric inequality $|u v| \leq|u|+|v|$.

Theorem 3. If $\phi \in \mathscr{R}\left(\mathbf{H}^{n}\right)$ is a nonzero function on $\mathbf{H}^{n}$ such that $\mathscr{L}^{-\frac{2 n+2}{4}} \phi \in L^{2}\left(\mathbf{H}^{n}\right)$ and $|\nabla \phi(u)| \leq C(1+|u|)^{-2 n-3-\epsilon}$, where constants $C, \epsilon>0$, then for $p=\frac{2 n+2}{2 n+1}$ and $q=2 n+2$, there exist constants $A_{q}, B_{p}>0$, such that

$$
A_{q}\left\|R_{n}(f)\right\|_{L^{q}\left(\mathbf{H}^{n}\right)} \leq\left\|g\left(R_{n} f\right)\right\|_{L^{q}\left(\mathbf{H}^{n}\right)} \leq B_{p}\|f\|_{L^{p}\left(\mathbf{H}^{n}\right)}
$$

for any $f \in L^{p}\left(\mathbf{H}^{n}\right)$.

To prove Theorem 3, we need the following several technique Lemmas.

Firstly, we consider the form of Fourier transform of $|x|^{\alpha-n}$, which is used to develop the boundedness of the singular convolution operator from $L^{p}\left(\mathbf{H}^{n}\right)$ to $L^{q}\left(\mathbf{H}^{n}\right)$, where $0<\alpha<n$. Now, we show the Fourier transform of a Guassian function. For $\varepsilon>0$, denote by $g_{\varepsilon}$ the Gaussian function on $\mathbb{R}^{n}$ given by

$$
g_{\varepsilon}(x)=\exp \left[-\pi|x|^{2} \varepsilon\right]
$$

for $x \in \mathbb{R}^{n}$. Then

$$
\widehat{g}_{\varepsilon}(k)=\varepsilon^{-n / 2} \exp \left[-\pi|k|^{2} / \varepsilon\right]
$$

By (16), we obtain the following lemma. 
Lemma 2. Let $c_{\alpha}:=\pi^{-\alpha / 2} \Gamma(\alpha / 2)(0<\alpha<n)$. Then, for any $s \in \mathbb{R}^{n}$,

$$
\Gamma(\alpha / 2) \int_{\mathbb{R}^{n}}|s|^{-\alpha} e^{2 \pi i \lambda s} d s=\pi^{\alpha / 2} c_{n-\alpha}|\lambda|^{\alpha-n} .
$$

Proof. Our starting point is the elementary formula

$$
c_{\alpha}|s|^{-\alpha}=\int_{0}^{\infty} \exp \left[-\pi|s|^{2} \varepsilon\right] \varepsilon^{\alpha / 2-1} d \varepsilon
$$

By Fubini's theorem, together with (18) and (16), we have,

$$
\begin{aligned}
\Gamma(\alpha / 2) \int_{\mathbb{R}}|s|^{-\alpha} e^{2 \pi i \lambda s} d s & =\pi^{\alpha / 2} \int_{0}^{\infty} \int_{\mathbb{R}} \exp \left[-\pi|s|^{2} \varepsilon\right] \varepsilon^{\alpha / 2-1} e^{2 \pi i \lambda s} d s d \varepsilon \\
& =\pi^{\alpha / 2} \int_{0}^{\infty} \varepsilon^{\alpha / 2-1} \int_{\mathbb{R}} \exp \left[-\pi|s|^{2} \varepsilon\right] e^{2 \pi i \lambda s} d s d \varepsilon \\
& =\pi^{\alpha / 2} \int_{0}^{\infty} \varepsilon^{-n / 2} \varepsilon^{\alpha / 2-1} \exp \left[-\pi|\lambda|^{2} / \varepsilon\right] d \varepsilon \\
& =\pi^{\alpha / 2} c_{n-\alpha}|\lambda|^{\alpha-n}
\end{aligned}
$$

Lemma 3. The estimate $\left\|R_{n} f\right\|_{q} \leq c\|f\|_{p}$ holds if and only if $p=\frac{2 n+2}{2 n+1}$ and $q=2 n+2$.

Proof. To show that the estimate $\left\|R_{n} f\right\|_{q} \leq c\|f\|_{p}$ holds we use an analytic families interpolation argument. We let

$$
T_{\alpha, n} f(z, t)=\Gamma(\alpha / 2) \int_{\mathbf{H}^{n}} T^{n} f\left(w, t+s+\frac{1}{2} \operatorname{Im}\langle z, w\rangle\right)|s|^{-\alpha} d s d w
$$

where $\alpha$ is complex parameter in the strip $0 \leq \operatorname{Re} \alpha \leq 1$. It is obvious that on the line $\operatorname{Re} \alpha=0$ the operator $T_{\alpha, n}$ is bounded from $L^{1}$ to $L^{\infty}$, while on the line $\operatorname{Re} \alpha=1$ a simple computation with (17) shows

$$
\mathscr{F}_{2} T_{\alpha, n} f(z, \lambda)=\pi^{\alpha / 2} c_{1-\alpha}|\lambda|^{\alpha-1}(2 \pi i \lambda)^{n} \mathscr{F} f(i \lambda z / 2, \lambda),
$$

and a modification of the proof of Theorem 1 shows that $T_{\alpha, n}$ is bounded from $L^{2}$ to $L^{2}$. The various $\Gamma$-factors are innocuous, so the Stein interpolation theorem yields the boundedness of $R_{n}$ from $L^{p}$ to $L^{q}$ for exactly $p=\frac{2 n+2}{2 n+1}, q=2 n+2$.

The necessity of proof is similar to that of [16, p. 387], the details being omitted.

The following lemma is just [12].

Lemma 4. If $\phi \in \mathscr{S}\left(\mathbf{H}^{n}\right)$ is a nonzero function on $\mathbf{H}^{n}$ such that $\mathscr{L}^{-\frac{2 n+2}{4}} \phi \in L^{2}\left(\mathbf{H}^{n}\right)$ and $|\nabla \phi(u)| \leq C(1+|u|)^{-2 n-3-\epsilon}$, where constants $C, \epsilon>0$, there exist constants $A_{p}, B_{p}>0$, such that

$$
A_{p}\|f\|_{L^{p}\left(\mathbf{H}^{n}\right)} \leq\|g(f)\|_{L^{p}\left(\mathbf{H}^{n}\right)} \leq B_{p}\|f\|_{L^{p}\left(\mathbf{H}^{n}\right)}
$$

for any $f \in L^{p}\left(\mathbf{H}^{n}\right)$. 
Proof. [Proof of Theorem 3] Let $f \in L^{p}\left(\mathbf{H}^{n}\right)$. For $p=\frac{2 n+2}{2 n+1}$ and $q=2 n+2$, by Lemma 3 , we know that $\left\|R_{n} f\right\|_{L^{q}\left(\mathbf{H}^{n}\right)} \leq c\|f\|_{L^{p}\left(\mathbf{H}^{n}\right)}$. From Lemma 4 , it follows that

$$
A_{q}\left\|R_{n} f\right\|_{L^{q}\left(\mathbf{H}^{n}\right)} \leq\left\|g\left(R_{n} f\right)\right\|_{L^{q}\left(\mathbf{H}^{n}\right)} \leq \widetilde{B_{q}}\left\|R_{n} f\right\|_{L^{q}\left(\mathbf{H}^{n}\right)} \leq B_{p}\|f\|_{L^{p}\left(\mathbf{H}^{n}\right)} .
$$

The proof is completed.

\section{The Poisson integral as a Radon transform on Šilov boundary $\partial U^{n+1}$}

Let $U^{n+1}$ be the generalized upper half-plane in $\mathbb{C}^{n+1}$,

$$
U^{n+1}=\left\{\left(z_{1}, \widetilde{z}\right) \in \mathbb{C}^{n+1}: \operatorname{Im} z_{1}>|\widetilde{z}|^{2}\right\}
$$

where $\widetilde{z}=\left(z_{2}, \cdots, z_{n+1}\right) \in \mathbb{C}^{n},|\widetilde{z}|^{2}=\sum_{j=2}^{n+1}\left|z_{j}\right|^{2}$; see, for example, [13].

For any $\alpha=\left(\alpha_{1}, \widetilde{\alpha}\right), \beta=\left(\beta_{1}, \widetilde{\beta}\right) \in U^{n+1}$, we consider the almost analytic extension of

$$
\rho(\alpha, \beta)=\frac{i}{2}\left(\bar{\beta}_{1}-\alpha_{1}\right)-\sum_{k=2}^{n+1} \alpha_{k} \bar{\beta}_{k} .
$$

In particular, when $\alpha=\beta$,

$$
\rho(\alpha, \alpha)=\rho(\alpha)=\operatorname{Im} \alpha_{1}-|\widetilde{\alpha}|^{2} .
$$

For $f$ holomorphic on the Siegel upper half-space $U^{n+1}$, we define

$$
\|f\|_{H^{2}}=\sup _{\rho>0}\left(\iint\left|f\left(\widetilde{z}, z_{1}+i|\widetilde{z}|^{2}+i \rho\right)\right|^{2} d|\widetilde{z}| d\left|z_{1}\right|\right)^{\frac{1}{2}} .
$$

Then we set

$$
H^{2}\left(U^{n+1}\right)=\left\{f: f \text { is holomorphic on } U^{n+1},\|f\|_{H^{2}}<\infty\right\},
$$

where $\rho$ is introduced on $U^{n+1}$.

For $F \in H^{2}\left(U^{n+1}\right)$, we let

$$
F_{\rho}(\widetilde{\alpha}, t)=F\left(\widetilde{\alpha}, t+i|\widetilde{\alpha}|^{2}+i \rho\right) .
$$

Definition 2. The Poisson-Szegö kernel $P$ is defined by

$$
P(\alpha, \beta):=\frac{|S(\alpha, \beta)|^{2}}{S(\alpha, \alpha)},
$$

where $S(\alpha, \beta)=\frac{(n+1) !}{4 \pi^{n+1}} \cdot \frac{1}{\rho(\alpha, \beta)^{n+1}}$ is Szegö kernel. 
Let the Poisson kernel $P$ be as in (20). Then, for any $f \in L^{2}\left(\partial U^{n+1}\right)$, we have

$$
F(\alpha)=\int_{\partial U^{n+1}} f(\beta) P(\alpha, \beta) d \sigma(\beta)
$$

where $d \sigma$ is a measure element of $\partial U^{n+1}$ (see [11]).

On the disk $D:|z|<1$, Helgason [9] proved that the Poisson integral and Radon transforms are equivalent. By [10, Proposition 2.5 and 2.6], we can obtain the following proposition.

Proposition 3. Let $F_{\rho}$ be as in (19). We assume that the Radon transform is the classical Poisson integral as in (21). Then the inverse formula is given by

$$
f(\widetilde{z}, t)=\lim _{\rho \rightarrow 0} F_{\rho}(\widetilde{z}, t)
$$

where $f(\widetilde{z}, t) \in L^{2}\left(\partial U^{n+1}\right)$.

\section{Acknowledgements}

The authors thank the anonymous referee for some expert comments and suggestions. The research of the second author was supported by National Natural Science Foundation of China (Grant Nos. 11471040, 11671414).

\section{References}

[1] H Afshari, H Marasi and H Aydi, Existence and uniqueness of positive solutions for boundary value problems of fractional differential equations, Filomat 31(2017), 26752682 .

[2] S Barker and S Salamon, Analysis on a generalized Heisenberg group, J. London Math. Soc. 28(1983) 184-192.

[3] H Elliott and L Michael, Analysis (second edition), Mathematical Gazette (1999).

[4] R Felix, Radon-Transformation auf nilpotenten Lie-Gruppen, Invent. Math. 112(1993) 413-443.

[5] D Geller and E M Stein, Estimates convolution operators on the Heisenberg group, Bull. Amer. Math. Soc. 267(1984) 99-103.

[6] J He, An inversion formula of the Radon transform on the Heisenberg group, Canad. Math. Bull. 47(2004) 389-397.

[7] $\mathrm{J} \mathrm{He}$ and $\mathrm{H} \mathrm{Liu}$, Inversion of the Radon transform associated with the classical domain of type one, Intern. J. Math. 16(2005) 875-887. 
[8] J He and H Liu, Admissible wavelets and inverse Radon transform associated with the affine homogenous Siegel domain of type two, Commun. Anal. Geom. 15(2007) 1-28.

[9] S Helgason, Integral Geometry and Radon Transforms, Springer New York (2011).

[10] A Korányi, The Poisson integral for generalized half-planes and bounded symmetric domains, Ann. of Math. 2(1965) 332-350.

[11] P Krantz, Explorations in Harmonic Analysis, Birkhäuser Boston (2009).

[12] H Liu and R Ma, Littlewood-Paley $g$-function on the Heisenberg Group, Acta Math. Sinica, English Series. 22(2006) 95-100.

[13] H Liu and L Peng, Admissible wavelets associated with the Heisenberg group, Pac. J. Math. 180(1997) 101-123.

[14] H Marasi H Piri and H Aydi, Existence and multiplicity of solutions for nonlinear fractional differential equations, J. Nonlinear Sci. Appl. 9(2016), 4639-4646.

[15] H Marasi, A solution of the new Caputo-Fabrizio fractonal KDV equation via stability, J. Math. Anal. 4(2017), 147-155.

[16] R Strichartz, $L^{p}$ harmonic analysis and Radon transforms on the Heisenberg group, J. Funct. Anal. 96(1991) 350-406.

[17] S Thangavelu, Harmonic Analysis on the Heisenberg Group, Birkhauser (1998). 